\title{
Monitoring the Ratio of two Normal Variables using Variable Sampling Interval EWMA control charts
}

\author{
Huu Du Nguyen ${ }^{1}$, Kim Phuc Tran*2, and Cédric Heuchenne ${ }^{3}$ \\ ${ }^{1}$ Division of Artificial Intelligence, Dong A University, Danang, \\ Vietnam \\ ${ }^{2}$ Ecole Nationale Supérieure des Arts et Industries Textiles, \\ GEMTEX Laboratory, BP 3032959056 Roubaix Cedex 1, France, \\ *Corresponding author. Email: kim-phuc.tran@ensait.fr \\ ${ }^{3}$ HEC Management School, University of Liège, Liège 4000, Belgium
}

November 15, 2019

\begin{abstract}
We investigate in this paper a new type of control chart called VSI EWMA-RZ by integrating the variable sampling interval feature (VSI) with the exponentially weighted moving average (EWMA) scheme to monitor the ratio of two normal random variables. Because the distribution of the ratio is skewed, we suggest designing two separated one-sided charts instead of one two-sided chart. A new coefficient is introduced allowing us to be free to choose a sampling interval provided that it optimizes the performance of the control chart. We also make a direct comparison between the VSI EWMA-RZ charts and standard EWMA-RZ control charts. The numerical simulations show that the proposed charts outperform the standard EWMA charts in detecting process shifts. An application is illustrated for the implementation of the VSI EWMA-RZ control charts in the food industry.
\end{abstract}

Keyword EWMA; VSI; Ratio distribution; Markov chain; Statistical Process Control.

\section{Introduction}

One important concern in manufacturing process of any factory or company is to maintain the quality of their products as committed in agreements. This makes the quality inspection becomes a key step in the production process. Of course, these companies should not wait until the goods are mass-produced to check the quality of each product or batches of products 
because it would be a huge waste if the batch are not up to standard. Instead, they wish to detect the abnormal products as well as the assignable causes that make the manufacturing out-of-control as soon as they occur. By this context, statistical process control (SPC) is an useful and efficient tool for monitoring the quality control process. Through control charts, the SPC helps manufactures to discover the abnormalities in the production line and fix this unexpected changes. As a consequence, the cost related to defective products will be reduced. Among the commonly used control charts, the exponentially weighted moving average (EWMA) chart type is proven to be effectively impressive in detecting small or moderate process shifts (Castagliola et al. ${ }^{1}$ ). The faster detection of small mean shifts of EWMA chart type compared to classical Shewhart chart type is because it utilizes the information embedded in the entire sequence of samples instead of using only the latest update of process measurements. A number of research on monitoring a process are carried out based on EWMA contral chart, see, Castagliola et al. ${ }^{2}$; Yeong et al. ${ }^{3}$; Castagliola et al. ${ }^{4}$ for more detail.

In a traditional way of designing a control chart, the sampling interval is supposed to be constant. The recent interests, however, convert into the use of control charts with variable sampling interval (VSI). That is to say, the sampling interval between two consecutive samples depends on the situation of the first sample. A short sampling interval is taken if a possible out-of-control situation of the plotted control statistic is indicated. Otherwise, a long sampling interval should be used. Since the amount of defective production is directly proportional to the time until detection, the overall quality of the process can be improved significantly by using the VSI control schemes. Various studies about the applications of VSI chart in recent years can be found in, for example, Yeong et al. ${ }^{3}$; Castagliola et al. ${ }^{5}$; Linna and Woodall ${ }^{6}$. It is important to consider that the VSI feature is just one among many other adaptive strategies to improve the performance of the original charts. Tagaras ${ }^{7}$ presented a comprehensive survey about the adaptive charts, including variable sample sizes (VSS), variable sampling intervals (VSI), variable control limit coefficients, and etc.

In reality, many processes require monitoring the ratio of two (or more) random variables. In food preparation industry, the correct balance of nutrition associated to the relative weights of two ingredients within a food recipe can affect the product specifications, see Celano et al. ${ }^{8}$. Also, it is important to strictly control the relative proportions of major ingredients of drugs in pharmaceutical industry to make sure that the drugs are safe and effective. In material manufacturing, a high performance of produced materials is ensured by keeping the percentage between their ingredients with additives in control. A general situation is mentioned in Celano and Castagliola ${ }^{9}$ when the change of a product quality characteristic measured 
before and after a chemical or physical reaction is represented by the dimensionless ratio. More examples of the use of the ratio of random variables can be seen in Celano et al. ${ }^{8}$, Tran et al. ${ }^{10}$ Tran et al. ${ }^{11}$. Nevertheless, there is not much SPC literature investigating monitoring the ratio of two variables since it is impossible to obtain an exact expression for the cumulative distribution function (c.d.f.) of the ratio while the probability density function (p.d.f.) is complicated, see Celano et al. ${ }^{8}$. Related to this topic, Öksoy et al. ${ }^{12}$ presented a set of guidelines for the implementation of Shewhart type control charts monitoring the ratio of two normal variables in the glass industry. The statistical properties of a Shewhart chart for individual measurements (the Shewhart-RZ control chart) are discussed by Celano and Castagliola ${ }^{13}$. They also extended this work to subgroups consisting of $n>1$ sample units where each of these units are allowed to change in size from one sample to another. Tran et al. ${ }^{10}$ incorporated Run Rules to the Shewhart-RZ control chart to make it more sensitive detecting small process shifts. The EWMA-RZ and the CUMSUM-RZ are investigated in Tran et al. ${ }^{14}$, Tran and Knoth ${ }^{15}$ and Tran et al. ${ }^{11}$, respectively. In 2016, Tran et al. ${ }^{16}$ firstly considered Shewhart-RZ chart in the presence of measurement errors. In a general case when quantity of each component in a mixture of multiple components can be modeled as a random vector, monitoring the compositional data is needed and Tran et al. ${ }^{17}$ provided a control chart for this kind of data.

Due to many practical applications, it is necessary to design an efficient control chart so that it is able to quickly detect the ratio shifts. In this study we suggest integrating the VSI feature into the EWMA chart on monitoring the ratio of population means of a bivariate normal distribution. In fact, the EWMA control charts for monitoring this ratio (denoted by EWMA-RZ control charts) have already discovered by Tran et al. ${ }^{14}$ and the authors have shown that their proposed charts are more sensitive to the process shifts than other existing charts in the literature. This design, however, is based on fixed sampling interval (FSI) type chart, which is demonstrated to be slower than VSI type chart in detecting process changes. Therefore, VSI EWMA-RZ presented in our research can be considered as a logical extension of the fixed scheme. The numerical results show that the VSI EWMA-RZ type chart outperforms significantly the original EWMA-RZ type chart.

The remainder of the paper is organized as follows: in Section 2, a brief review of the sample distribution of the ratio of two normal variables is recalled. The two one-sided VSI EWMA-RZ charts are defined in Section 3 , while formula for average time to signal $(A T S)$ is presented in Section 4 using a Markov Chain-based approach. Section 5 is devoted to optimizing design parameters. An illustrative example from the real industrial data is provided to show the implementation of VSI EWMA-RZ control charts in 
Section 6. Some concluding remarks are given in Section 7.

\section{A brief review of the distribution of the sample of the ratio}

Let $\mathbf{W}=(X, Y)^{T}$ is a bivariate normal random vector with mean vector

$$
\boldsymbol{\mu}_{\boldsymbol{W}}=\left(\begin{array}{c}
\mu_{X} \\
\mu_{Y}
\end{array}\right)
$$

and variance-covariance matrix

$$
\boldsymbol{\Sigma}_{\boldsymbol{W}}=\left(\begin{array}{cc}
\sigma_{X}^{2} & \rho \sigma_{X} \sigma_{Y} \\
\rho \sigma_{X} \sigma_{Y} & \sigma_{Y}^{2}
\end{array}\right)
$$

In this definition, $\rho$ is the correlation coefficient between two components $X$ and $Y ; \mu_{X}$ and $\sigma_{X}$ are the mean and the variance of $X$ while $\mu_{Y}$ and $\sigma_{Y}$ are the mean and the variance of $Y$. The coefficients of varation of two variables $X$ and $Y$ are then defined by $\gamma_{X}=\frac{\sigma_{X}}{\mu_{X}}$ and $\gamma_{Y}=\frac{\sigma_{Y}}{\mu_{Y}}$; the standard-deviation ratio between them is $\omega=\frac{\sigma_{X}}{\sigma_{Y}}$.

The ratio of $X$ to $Y, Z=\frac{X}{Y}$, is in our interest. Although there are a number of study about the distribution of the ratio of two normal variables, for example Geary ${ }^{18}$ and Hayya et al. ${ }^{19}$, Cedilnik et al. ${ }^{20}$ and Pham-Gia et al. ${ }^{21}$, the closed-form expression for its distribution has not been given yet. Instead, the distribution of $Z$ can be well approximated by using an approach similar to the one suggested by Geary ${ }^{18}$, Hayya et al. ${ }^{19}$ and Celano and Castagliola ${ }^{13}$. In this paper, we will resort to a following approximation of the c.d.f. of $Z$ :

$$
F_{Z}\left(z \mid \gamma_{X}, \gamma_{Y}, \omega, \rho\right) \simeq \Phi\left(\frac{A}{B}\right)
$$

where $\Phi($.$) is the c.d.f. of the standard normal distribution; A$ and $B$ are functions of $z, \gamma_{X}, \gamma_{Y}, \omega$ and $\rho$, i.e.

$$
\begin{aligned}
A & =\frac{z}{\gamma_{Y}}-\frac{\omega}{\gamma_{X}}, \\
B & =\sqrt{\omega^{2}-2 \rho \omega z+z^{2}} .
\end{aligned}
$$

The p.d.f. of $Z$ can be approximated by

$$
f_{Z}\left(z \mid \gamma_{X}, \gamma_{Y}, \omega, \rho\right) \simeq\left(\frac{1}{B \gamma_{Y}}-\frac{(z-\rho \omega) A}{B^{3}}\right) \times \phi\left(\frac{A}{B}\right)
$$

where $\phi($.$) is the p.d.f. of the standard normal distribution, see Celano$ and Castagliola ${ }^{13}$. In Figure 1, the p.d.f of $Z$ is displayed with different 
Figure 1: Approximate density function of two random normal variables

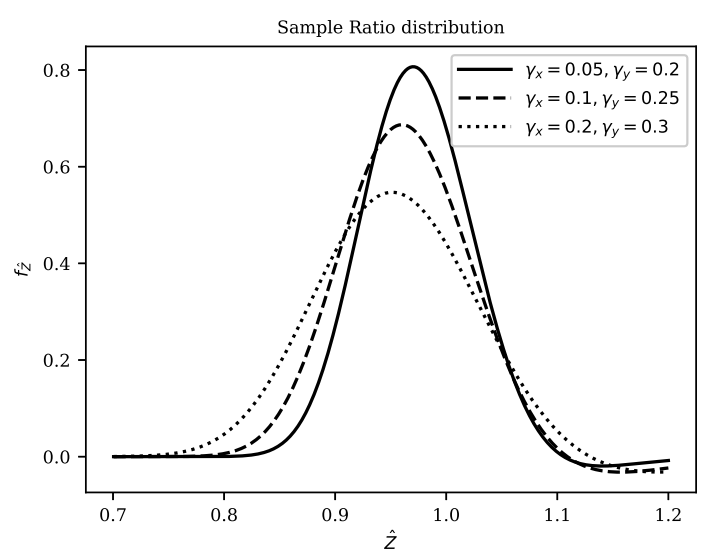

parameters. The inverse distribution function (i.d.f.) of $Z$ is obtained by inverting the c.d.f. of $Z$ in (3), leading to

$$
F_{Z}^{-1}\left(p \mid \gamma_{X}, \gamma_{Y}, \omega, \rho\right) \simeq \begin{cases}\frac{-C_{2}-\sqrt{C_{2}^{2}-4 C_{1} C_{3}}}{2 C_{1}} & \text { if } p \in(0,0.5], \\ \frac{-C_{2}+\sqrt{C_{2}^{2}-4 C_{1} C_{3}}}{2 C_{1}} & \text { if } p \in[0.5,1),\end{cases}
$$

where $C_{1}, C_{2}$ and $C_{3}$ are functions of $p, \gamma_{X}, \gamma_{Y}, \omega$ and $\rho$, i.e.

$$
\begin{aligned}
& C_{1}=\frac{1}{\gamma_{Y}^{2}}-\left(\Phi^{-1}(p)\right)^{2}, \\
& C_{2}=2 \omega\left(\rho\left(\Phi^{-1}(p)\right)^{2}-\frac{1}{\gamma_{X} \gamma_{Y}}\right), \\
& C_{3}=\omega^{2}\left(\frac{1}{\gamma_{X}^{2}}-\left(\Phi^{-1}(p)\right)^{2}\right),
\end{aligned}
$$

and where $\phi^{-1}($.$) stands for the i . d . f$. of the standard normal distribution.

The ratio $Z$ is monitored in practice by firstly collecting a sample of $n$ independent couples $\left\{\mathbf{W}_{i, 1}, \mathbf{W}_{i, 2}, \ldots, \mathbf{W}_{i, n}\right\}$ at each sampling period $i=$ $1,2, \ldots$, where $\mathbf{W}_{i, j}=\left(X_{i, j}, Y_{i, j}\right)^{T} \sim N\left(\boldsymbol{\mu}_{\mathbf{W}, i}, \boldsymbol{\Sigma}_{\mathbf{W}, i}\right), j=1, \ldots, n$. That is to say, each $\mathbf{W}_{i, j}$ is a bivariate normal random vector with mean vector

$$
\boldsymbol{\mu}_{\mathbf{W}, i}=\left(\begin{array}{c}
\mu_{X, i} \\
\mu_{Y, i}
\end{array}\right)
$$

and variance-covariance matrix

$$
\boldsymbol{\Sigma}_{\mathbf{W}, i}=\left(\begin{array}{cc}
\sigma_{X, i}^{2} & \rho \sigma_{X, i} \sigma_{Y, i} \\
\rho \sigma_{X, i} \sigma_{Y, i} & \sigma_{Y, i}^{2}
\end{array}\right) .
$$


Following the discussion in Celano and Castagliola ${ }^{13}$, some assumptions are supposed for the collected samples. The first assumption is about the linear relationship between the mean and the standard deviation of $X$ and $Y$. Because many quality characteristics have a dispersion proportional to the population mean, it is practical to use a known and constant coefficient of variation of both $X$ and $Y$. That means the equations $\sigma_{X, i}=\gamma_{X} \times \mu_{X, i}$ and $\sigma_{Y, i}=\gamma_{Y} \times \mu_{Y, i}$ hold for every $i$. The second one is that the ratio $\frac{\mu_{X, i}}{\mu_{Y, i}}$ is equal to $z_{0}$ for every samples provided that the process is in-control. Also, the sample units are permitted to be free to change from sample to sample, i.e. it is not necessary to impose the constraints $\boldsymbol{\mu}_{\mathbf{W}, i}=\boldsymbol{\mu}_{\mathbf{W}, k}$ and $\boldsymbol{\Sigma}_{\mathbf{W}, i}=\boldsymbol{\Sigma}_{\mathbf{W}, k}$ for $i \neq k$. Then, the observed statistic is

$$
\hat{Z}_{i}=\frac{\hat{\mu}_{X, i}}{\hat{\mu}_{Y, i}}=\frac{\bar{X}_{i}}{\bar{Y}_{i}}=\frac{\sum_{j=1}^{n} X_{i, j}}{\sum_{j=1}^{n} Y_{i, j}}, i=1,2, \ldots
$$

Based on the c.d.f. and the i.d.f. of $Z$, Celano and Castagliola ${ }^{13}$ deduced the c.d.f. and the i.d.f. of $\hat{Z}_{i}$ as follows:

$$
\begin{gathered}
F_{\hat{Z}_{i}}\left(z \mid n, \gamma_{X}, \gamma_{Y}, z_{0}, \rho_{0}\right)=F_{Z}\left(z \mid \frac{\gamma_{X}}{\sqrt{n}}, \frac{\gamma_{Y}}{\sqrt{n}}, \frac{z_{0} \gamma_{X}}{\gamma_{Y}}, \rho_{0}\right), \\
F_{\hat{Z}_{i}}^{-1}\left(p \mid n, \gamma_{X}, \gamma_{Y}, z_{0}, \rho_{0}\right)=F_{Z}^{-1}\left(p \mid \frac{\gamma_{X}}{\sqrt{n}}, \frac{\gamma_{Y}}{\sqrt{n}}, \frac{z_{0} \gamma_{X}}{\gamma_{Y}}, \rho_{0}\right) .
\end{gathered}
$$

\section{Implementation of VSI EWMA-RZ control charts}

Since the distribution of $Z$ is non-symmetric, we investigate in this paper two separate one-sided VSI EWMA-RZ control charts. We firstly recall FSI EWMA-RZ control charts suggested in Tran et al. ${ }^{14}$ as follows.

- An upward EWMA chart for detecting the increase in the ratio that monitors the statistic

$$
Y_{i}^{+}=\max \left(z_{0},\left(1-\lambda^{+}\right) Y_{i-1}^{+}+\lambda^{+} \hat{Z}_{i}\right)
$$

with the single upper control limit is $U C L^{+}=H_{U} \times z_{0}$ and the initial value $Y_{0}^{+}=z_{0}$. The corresponding lower control limit is $L C L^{+}=z_{0}$.

- A downward EWMA chart for detecting the decrease in the ratio that monitors the statistic

$$
Y_{i}^{-}=\min \left(z_{0},\left(1-\lambda^{-}\right) Y_{i-1}^{-}+\lambda^{-} \hat{Z}_{i}\right)
$$

with the single lower control limit is $L C L^{-}=H_{D} \times z_{0}$ and the initial value $Y_{0}^{-}=z_{0}$. The corresponding upper control limit is $U C L^{-}=z_{0}$. 
In this definition, $\lambda^{+} \in(0,1]$ and $H_{U}>1\left(\lambda^{-} \in(0,1]\right.$ and $\left.H_{D}<1\right)$ are the smoothing and chart parameters of the upward (downward) chart, respectively. It is proven that the EWMA-RZ chart is sensitive to small and moderate shifts. In comparison with others, however, it is less sensitive toward large shifts. The incorporation of the VSI feature into EWMA-RZ chart is then expected to improve this insensitivity toward large shifts and increase the performance of the charts.

In the VSI EWMA- $R Z$ control charts, the control limits $U C L^{+}=H_{U} \times$ $z_{0}$ and $L C L^{-}=H_{D} \times z_{0}$ are hold the same as those in original FSI charts. The difference is that the sampling interval, i.e., the time between two successive samples $Z_{i}$ and $Z_{i+1}$, is allowed to vary based on the current value of $Y_{i}^{+}$or $Y_{i}^{-}$. For the upward (downward) control chart, a longer sampling interval, $h_{L}$, is used when the control statistic falls within region $\left[z_{0}, U W L^{+}\right]\left(\left[L W L^{-}, z_{0}\right]\right)$, in which

- $U W L^{+}=z_{0}+R_{U}\left(U C L^{+}-z_{0}\right)$ is the upper warning limit,

- $L W L^{-}=z_{0}-R_{D}\left(z_{0}-L C L^{-}\right)$is the lower warning limit.

Similarly, the short sampling interval, $h_{S}$, is used when the control statistic falls within the region $\left[U W L^{+}, U C L^{+}\right]$(upward case) and $\left[L C L^{-}, L W L^{-}\right]$ (downward case). Moreover, an out-of-control signal is given at time $i$ if $Z_{i}^{+}>U C L^{+}$for the upward chart or $Z_{i}^{-}<L C L^{-}$for downward chart. Thus, in VSI EWMA- $R Z$ scheme, the control interval is separated into three regions: the "safe" or central region, the warning region and the outof-control region. Figure 2 illustrates a graphical view of the operation of the upward VSI EWMA-RZ chart. The warning limit $U W L^{+}$or $L W L^{-}$are defined through the new parameters $R_{U} \in(0,1)$ or $\left(R_{D} \in(0,1)\right)$. These new parameters are called the warning limit coefficients. They represent the relation between warning region and safe region in the sense that the smaller are the values of $R_{U}$ or $R_{D}$, the smaller is the "safe region" compared to warning region.

\section{Design of optimal VSI EWMA-RZ control charts}

Suppose that the occurrence of an assignable cause shifts the in-control ratio $z_{0}$ to $z_{1}=\tau \times z_{0}$, where $\tau>0$ is the shift size. The values $\tau<1$ correspond to the decrease of the nominal ratio $z_{0}$, while the values $\tau>1$ represent the increase of the nominal ratio $z_{0}$. When the process shifts to the out-ofcontrol condition, the coefficient of correlation can be shifted from $\rho=\rho_{0}$ to $\rho=\rho_{1}$. We firstly discuss in this Section a method to compute the average of the zero-state time to signal for the two one-sided VSI EWMA-RZ control charts. The ATS counts the expected time before a control chart signals an out-of-control condition after the occurrence of an assignable cause or the 
Figure 2: Three regions of the upward VSI EWMA-RZ control charts

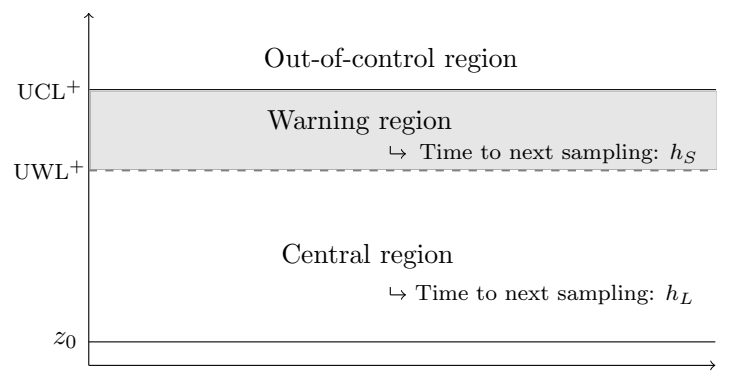

issue of a false alarm. When a process runs in-control, it is denoted by $A T S_{0}$; otherwise, it is denoted by $A T S_{1}$. By its meaning, it is desirable to design a chart with smaller $A T S_{1}$ while the $A T S_{0}$ is still the same in comparison with others. For a FSI model, the ATS is a multiple of the $A R L$ since the sampling interval $h_{F}$ is fixed, namely,

$$
A T S^{\mathrm{FSI}}=h_{F} \times A R L^{\mathrm{FSI}} .
$$

For a VSI model, the $A T S$ is defined as:

$$
A T S^{\mathrm{VSI}}=E(h) \times A R L^{\mathrm{VSI}} .
$$

where $E(h)$ is the average sampling interval.

The ATS measure is calculated using the discrete Markov chain approach proposed by Brook and Evans ${ }^{22}$. The method is performed by partitioning the control interval into a finite set of $p$ sub-intervals corresponding to $p+2$ states of the Markov chain. The width of each sub-interval is $2 \delta$ where $\delta=\frac{U C L^{+}-1}{2 p}\left(\delta=\frac{1-L C L^{-}}{2 p}\right)$. The value of $p$ is chosen so that each midpoint $H_{j}, j=1, \ldots, p$ can be considered as the representative of the sub-interval $\left(H_{j}-\delta, H_{j}+\delta\right]$. Figure 3 illustrates this subdivision for upward chart (without loss of generality, in the remaining part of this paper we suppose that $z_{0}=1$.) Among $p+2$ states of Markov chain in the Figure 3 , the state 0 is corresponding to the line $H_{0}$, the in-control transient state $j$ corresponds to the $j^{\text {th }}$ sub-intervals, $j=1, . ., p$ while the state $p+2$ is out-of-control or absorbing state. If the statistic $Y_{i}^{+}$or $Y_{i}^{-}$fall into the sub-interval $j$, the Markov chain is in the transient sate $j$ for sample $i$; if not, the chain reaches absorbing state. The transition probability matrix $\mathbf{P}$ of this discrete 
Figure 3: Sub-division of the control interval of upward chart into $p$ subintervals of width $2 \delta$.

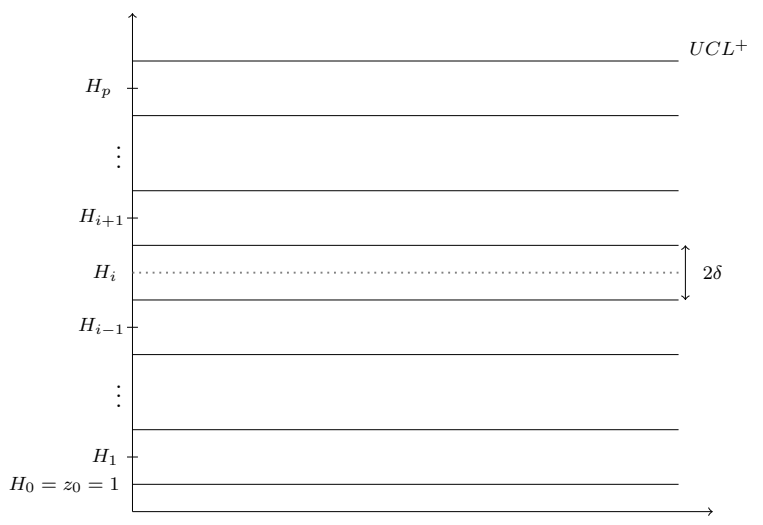

Markov chain is

$$
\mathbf{P}=\left(\begin{array}{cc}
\mathbf{Q} & \mathbf{r} \\
\mathbf{0}^{T} & 1
\end{array}\right)=\left(\begin{array}{ccccc}
Q_{0,0} & Q_{0,1} & \ldots & Q_{0, p} & r_{0} \\
Q_{1,0} & Q_{1,1} & \ldots & Q_{1, p} & r_{1} \\
\vdots & \vdots & & & \vdots \\
Q_{p, 0} & Q_{p, 1} & \ldots & Q_{p, p} & r_{p} \\
0 & 0 & \ldots & 0 & 1
\end{array}\right)
$$

In the above formula, $\mathbf{Q}$ is the $(p+1, p+1)$ matrix of transient probabilities, $\mathbf{0}=(0,0, \ldots, 0)^{T}$ and the $(p+1)$ vector $\mathbf{r}$ satisfies $\mathbf{r}=(\mathbf{1}-\mathbf{Q 1})$ (row probabilities must sum to 1$)$ with $\mathbf{1}=(1,1, \ldots, 1)^{T}$. Denote $\mathbf{q}$ the $(p+1,1)$ vector of initial probabilities associated with the $p+1$ transient states, i.e., $\mathbf{q}=\left(q_{0}, q_{1}, \ldots, q_{p}\right)^{T}$. The generic element $Q_{i, j}, i=0,1, \ldots, p$, of the matrix $\mathbf{Q}$ of transient probabilities is defined by

- if $j=0$,

$$
\begin{aligned}
& \text { for the upward chart : } Q_{i, 0}=F_{\hat{Z}_{i}}\left(\frac{1-\left(1-\lambda^{+}\right) H_{i}}{\lambda^{+}} \mid n, \gamma_{X}, \gamma_{Y}, z_{1}, \rho_{1}\right) \text {, } \\
& \text { for the downward chart : } Q_{i, 0}=1-F_{\hat{Z}_{i}}\left(\frac{1-\left(1-\lambda^{-}\right) H_{i}}{\lambda^{-}} \mid n, \gamma_{X}, \gamma_{Y}, z_{1}, \rho_{1}\right)
\end{aligned}
$$

- if $j=1,2, \ldots, p$, for both cases,

$$
\begin{aligned}
Q_{i, j}=F_{\hat{Z}_{i}}( & \left.\frac{H_{j}+\delta-(1-\lambda) H_{i}}{\lambda} \mid n, \gamma_{X}, \gamma_{Y}, z_{1}, \rho_{1}\right) \\
& -F_{\hat{Z}_{i}}\left(\frac{H_{j}-\delta-(1-\lambda) H_{i}}{\lambda} \mid n, \gamma_{X}, \gamma_{Y}, z_{1}, \rho_{1}\right),
\end{aligned}
$$


where $F_{\hat{Z}_{i}}($.$) is the c.d.f. of \hat{Z}_{i}$ as defined in (3) while $\lambda$ is either $\lambda^{+}$or $\lambda^{-}$, corresponding to upward or downward case. Also, the vector q of initial probabilities, concerning the zero-state condition, is equal to $\mathbf{q}=(1,0, \ldots, 0)$.

The formula of $A T S$ is suggested by Saccucci et al. ${ }^{23}$ as

$$
A T S=\mathbf{q}^{T}(\mathbf{I}-\mathbf{Q})^{-1} \mathbf{g}
$$

where $\mathbf{I}$ is the identity matrix, $\mathbf{g}$ is the vector of sampling intervals corresponding to the discretized states of the Markov chain. The element $g_{j}$ of the vector $\mathbf{g}$ is the sampling interval when the control statistic is in the state $j$ (denoted by $H_{j}$ ). That is to say,

$$
g_{j}=\left\{\begin{array}{ll}
h_{S} & \text { if } H_{j} \in\left[U W L^{+}, U C L^{+}\right] \text {or } H_{j} \in\left[L C L^{-}, L W L^{-}\right] \\
h_{L} & \text { if } H_{j} \in\left[z_{0}, U W L^{+}\right] \text {or } H_{j} \in\left[L W L^{-}, z_{0}\right]
\end{array} .\right.
$$

From the equations (19) and (23), the expected sampling interal $E(h)$ is calculated by

$$
E(h)=\frac{\mathbf{q}^{T}(\mathbf{I}-\mathbf{Q})^{-1} \mathbf{g}}{\mathbf{q}^{T}(\mathbf{I}-\mathbf{Q})^{-1} \mathbf{1}}
$$

where the denominator in (25) is the formula for $A R L$ as in Brook and Evans $^{22}$.

It is quite often that the constant sampling interval in FSI control charts is supposed to be time unit, i.e. $h_{F}=1$. As a result, $A T S_{0}^{F S I}=A R L_{0}$. A fair comparison between two types of control charts is made by constraining the same in-control values of both average time to signal $A T S_{0}$ and average sampling interval $E_{0}(h)$. Saccucci et al. ${ }^{23}$ insisted that using two sampling intervals is optimal for detecting a specified shift of the process target value or any control scheme that can be represented as a Markov chain. A fixed couple $\left(h_{S}, h_{L}\right)$ is then typically used which is chosen from a suggested list as in Yeong et al. ${ }^{3}$ and Castagliola et al. ${ }^{5}$. Because $h_{S}$ represents the shortest feasible time interval between subgroups from the process Castagliola et al. ${ }^{1}$, it is quite reasonable to fix the value of $h_{S}$ according to the manufacturing conditions. However, it seems not practical to fix the value of $h_{L}$. Once the control statistic drops into central region, the process is still 'in safe' and the next sampling interval can be free to choose as long as it does not affect the performance of the chart. For this reason, we suggest to fix the warning limit coefficient $R_{U}\left(R_{D}\right)$. Once the control limit is defined, the value of warning limit is determined given the value of $R_{U}\left(R_{D}\right)$. The optimal parameters now is $\left(\lambda^{+}, H_{U}, h_{L}\right)$ for upward chart and $\left(\lambda^{-}, H_{D}, h_{L}\right)$ for downward chart, using fixed values of $h_{S}$. Given the size $\tau$, we find out the combination $\left(\lambda^{+*}, H_{U}^{*}, h_{L}^{*}\right)$ or $\left(\lambda^{-*}, H_{D}^{*}, h_{L}^{*}\right)$ such that 
- for downward chart,

$$
\left(\lambda^{+*}, H_{U}^{*}, h_{L}^{*}\right)=\underset{\left(\lambda^{+}, H_{U}, h_{L}\right)}{\operatorname{argmin}} \operatorname{ATS}\left(n, \lambda^{+}, H_{U}, R_{U}, \rho, \gamma_{X}, \gamma_{Y}, \tau, h_{L}, h_{S}\right)
$$

subject to the constraint

$$
\left\{\begin{array}{l}
A T S\left(n, \lambda^{+*}, H_{U}^{*}, R_{U}, \rho, \gamma_{X}, \gamma_{Y}, \tau=1, h_{L}^{*}, h_{S}\right)=A T S_{0} \\
E_{0}(h)=1
\end{array} ;\right.
$$

- for upward chart,

$$
\left(\lambda^{-*}, H_{D}^{*}, h_{L}^{*}\right)=\underset{\left(\lambda^{-}, H_{D}, h_{L}\right)}{\operatorname{argmin}} \operatorname{ATS}\left(n, \lambda^{-}, H_{D}, R_{D}, \rho, \gamma_{X}, \gamma_{Y}, \tau, h_{L}, h_{S}\right)
$$

subject to the constraint

$$
\left\{\begin{array}{l}
A T S\left(n, \lambda^{-*}, H_{D}^{*}, R_{D}, \rho, \gamma_{X}, \gamma_{Y}, \tau=1, h_{L}^{*}, h_{S}\right)=A T S_{0} \\
E_{0}(h)=1
\end{array} .\right.
$$

\section{Numerical results and comparison}

We firstly compute in this Section the optimal parameters $\left(\lambda^{+*}, H_{U}^{*}, h_{L}^{*}\right)$ for upward chart or $\left(\lambda^{-*}, H_{D}^{*}, h_{L}^{*}\right)$ for downward chart that minimize $A T S_{1}$ subject to constraints $A T S_{0}=200$ and $E_{0}(h)=1$. The optimizing method is applied based on a number of following scenarios of parameters:

- $\gamma_{X} \in\{0.01,0.2\}, \gamma_{Y} \in\{0.01,0.2\}$;

- $\rho_{0} \in\{0.0, \pm 0.4, \pm 0.8\}$;

- $n \in\{1,5,10,15\}$;

- $\tau \in\{0.9,0.95,098,0.99,1.01,1.02,1.05,1.1\}$;

- $R_{U}, R_{D} \in\{0.1,0.2,0.3,0.5\}$;

- $h_{S} \in\{0.1,0.5\}$.

These scenarios are supposed for a desire of covering a large range of possible situations of parameters. For example, the values 0.01 and 0.2 of $\gamma_{X}$ ( or $\gamma_{Y}$ ) represent the small and large variation of $X$ (or $Y$ ). The values vary from -0.8 to 0.8 of $\rho_{0}$ is to describe the strong or weak, negative or positive correlation between $X$ and $Y$. Similarly, the value $n=1$ or $n=5$ is corresponding to small sample size while $n=15$ is for large sample size. The value $R_{U}=0.1\left(R_{D}=0.1\right)$ represents very small central region, the value $R_{U}=0.5\left(R_{D}=0.5\right)$ represents an equal central region compared to 
the warning region. The value $\tau=0.9$ is for the large decrease of process shift and $\tau=1.01$ is for the small increase of process shift. Finally, the value of $h_{S}$ is recommended by Reynolds and Arnold Reynolds and Arnold ${ }^{24}$.

After computing these optimal parameters, the out-of-control value of $A T S$ are numerically obtained given a specific shift size $\tau$. The obtained results are shown in Tables 1-8. The corresponding parameters in FSI EWMA$\mathrm{RZ}$ chart are also provided in column titled FSI. These tables show only the results for the case the correlation coefficient between two variables does not depend on the condition of the process, namely $\rho_{1}=\rho_{0}$. The numerical results corresponding to the different values of $\rho_{1}$ are not presented for the sake of brevity but are available upon request from authors. Some remarks can be drawn as follows.

- In general, the VSI EWMA-RZ control charts outperform the standard EWMA-RZ control charts in detecting the process shifts. The values of $A R L_{1}$, representing the performance of EWMA-RZ charts are relatively larger than those the values of $A T S_{1}$, representing the VSI EWMA-RZ charts performance, in most cases observed. For example, with $\gamma_{X}=\gamma_{Y}=0.2, \rho_{0}=-0.8, R_{U}=0.1, h_{S}=0.1, \tau=1.05$ and $n=15$ in Table $4, A R L_{1}=20.1$ while $A T S_{1}=10.6$.

- The performance of the proposed charts is strongly influenced by the coefficient of variation (CV) $\gamma_{X}$ and $\gamma_{Y}$ of both two variables. For cases where the two variables have small CV, the VSI EWMA-RZ chart are very sensitive to the ratio shifts. This sensitivity decreases when the CV of two variables increases. Taking the case where $h_{S}=$ $0.5, n=1, R_{D}=0.2, \rho=-0.4$ and $\tau=0.98$ as an example; we have $A T S_{1}=4.3$ for $\gamma_{X}=\gamma_{Y}=0.01$ (Table 1) and $A T S_{1}=20.6$ for $\gamma_{X}=0.01, \gamma_{Y}=0.2$ (Table 6$)$.

- Given the value of others, the values of $A T S_{1}$ and $H_{U}^{*}\left(H_{D}^{*}\right)$ vary with $\rho$. Particularly, the increase of the correlation $\rho$ makes the VSI EWMA-RZ charts performance better. With respect to the control intervals, it generally reduces the values of $H_{U}^{*}\left(H_{D}^{*}\right)$. For example, with $n=1, \gamma_{X}=0.01, \gamma_{Y}=0.2, \tau=0.99, R_{D}=0.5$ and $h_{S}=0.1$, we have $\left(A T S_{1}, H_{D}^{*}\right)=(134.7,0.985)$ for $\rho=-0.4$ while $\left(A T S_{1}, H_{D}^{*}\right)=(131.3,0.953)$ for $\rho=0.8$ (Table 5).

- The chart efficiency is also affected by $h_{S}$. The values of $A T S_{1}$ in the left block (corresponding to $h_{S}=0.1$ ) are always smaller than those in the right block (corresponding to $h_{S}=0.5$ ). In addition the smaller value of $h_{S}$ also leads to the larger value of $h_{L}^{*}$. For example, with $\gamma_{X}=0.2, \gamma_{Y}=0.01, n=1, R_{D}=0.2, \rho=0.4$ and $\tau=0.98$ in Table 7, 
we obtain $A T S_{1}=83.0$ and $h_{L}^{*}=2.132$ for $h_{S}=0.1$, but $A T S_{1}=91.9$ and $h_{L}^{*}=1.628$ for $h_{S}=0.5$.

- The predetermined value of warning limit coefficient $R_{U}\left(R_{D}\right)$ has opposite effect on the VSI EWMA-RZ control charts performance and the sampling interval in central region. Generally, the increase of $R_{U}\left(R_{D}\right)$ reduces the value of $h_{L}^{*}$ but the value of $A T S_{1}$. For example, we have $\left(h_{L}^{*}, A T S_{1}\right)=(2.172,122.6)$ for $R_{U}=0.1$ and $\left(h_{L}^{*}, A T S_{1}\right)=$ $(1.119,127.4)$ for $R_{U}=0.5$ when $\gamma_{X}=0.2, \gamma_{Y}=0.1, \rho=0.8, n=$ $1, h_{S}=0.5$ and $\tau=1.01$ in Table 7 . This is because the values closer to 0 of $R_{U}\left(R_{D}\right)$ correspond to the larger warning region in comparison with central region. As a consequence, the sample points corresponding to the trend towards outside of the control interval are detected faster and the VSI EWMA-RZ control charts is more sensitive to the process shift.

The above analysis is based on the deterministic values of $\tau$. However, according to the discussion of Castagliola et al. ${ }^{5}$, there are a number of reasonable explanations for the difficulty of predicting an exact value of the shift size. In such situations, it is suggested to use an expected average time to signal $(E A T S)$ as an alternative measure of the $A T S$. That is

$$
E A T S=\int_{a}^{b} A T S \times f_{\tau}(\tau) \mathrm{d} \tau
$$

where $[a, b]$ is the predicted interval of the shift size $\tau$ and $f_{\tau}(\tau)$ is density function of $\tau$. In the case there is no information about $\tau$, an uniform distribution can be applied, namely $f_{\tau}(\tau)=\frac{1}{b-a}$. The new optimal triples $\left(\lambda^{+*}, H_{U}, h_{L}^{*}\right)$ or $\left(\lambda^{-*}, H_{D}, h_{L}^{*}\right)$ is now to minimize the out-of-control $E A T S_{1}$ for a given in-control $A T S_{0}$ and subject to the constraints $E_{0}(h)=1$ and $A T S_{0}=200$. This procedure consists of two following steps:

1. Solve the potential triples $\left(\lambda^{+}, H_{U}, h_{L}\right)$ or $\left(\lambda^{-}, H_{D}, h_{L}\right)$ such that $A T S=A T S_{0}$ and $E_{0}(h)=1$.

2. Choose from these triples the one $\left(\lambda^{+*}, H_{U}^{*}, h_{L}\right)$ or $\left(\lambda^{-*}, H_{D}^{*}, h_{L}\right)$ corresponding to the smallest value of the EATS.

Table 9 - 12 present the values of the EATS corresponding to two different ranges of shifts, $[a, b]=[0.9,1)$ (decreasing shift sizes) and $[a, b]=(1,1.1]$ (increasing cases). For comparison purpose we also compute the values of $E A R L_{1}$, the control limits and the chart parameters $H_{U}$ or $H_{D}$ of FSI EWMA chart. The obtained results in these tables show a similar trend as for the deterministic shift size discussed above. Moreover, they also reveal a somewhat more overview. 
- In general, the smaller values of warning limit coefficient results in the better efficiency of proposed chart. In most situations observed, the smallest value of $E A T S_{1}$ is corresponding to the smallest value of $R_{U}\left(R_{D}\right)$, which is 0.1 in this paper. When $\gamma_{X}$ and $\gamma_{Y}$ are both small, however, the best performance of VSI EWMA-RZ chart is obtained corresponding to $R_{U}=R_{D}=0.3$, see Table 9 .

- When $\gamma_{X}=\gamma_{Y}$ and they are both small, the value of $E A T S_{1}$ for both cases are approximate. For example, in Table 9 when $\left(\gamma_{X}, \gamma_{Y}\right)=$ (0.01,0.01), $n=1, \rho=0.4, h_{S}=0.1$, and $R_{U}=R_{D}=0.3$, it results $E A T S_{1}=1.8$ for both the increasing and decreasing cases. In other situations, the symmetrical performance does not hold.

\section{Illustrative example}

In this Section, using the same dataset simulating a real quality control problem from the food industry as shown in Celano et al. ${ }^{8}$, we present an illustrative example of implementation of the VSI EWMA-RZ chart. A food company produces a muesli brand recipe which is a mixture of several ingredients, involving two main components pumpkin seeds and flaxseeds with added spices. The requirements of food's nutrient composition is maintained by ensuring the equal weights of "pumpkin seeds" and "flaxseeds", which is fixed at $p_{p}=p_{f}=0.1$. The brand boxes are manufactured in different sizes to meet the need of customers. Because of the problems occurring at the dosing machine, the manufacturers wish to perform on-line SPC monitoring at regular intervals $i=1,2, \ldots$ to check deviations from the in-control ratio $z_{0}=\frac{\mu_{p, i}}{\mu_{f, i}}=\frac{0.1}{0.1}=1$ for every size of boxes, where $\mu_{p, i}$ and $\mu_{f, i}$ denote the mean weights for "pumpkin seeds" and "flaxseeds", respectively. Every 30 minutes, the quality practitioner collects a sample of $n=5$ boxes to implement an upward VSI EWMA-RZ control chart. The sample average weights $\bar{W}_{p, i}=\frac{1}{n} \sum_{j=1}^{n} W_{p, i, j}$ and $\bar{W}_{f, i}=\frac{1}{n} \sum_{j=1}^{n} W_{f, i, j}$ are kept account by separating the "pumpkin seeds" and "flaxseeds" from the muesli mixture filling each box using a mechanical procedure in the quality control laboratory. The ratio $\hat{Z}_{i}=\frac{\bar{W}_{p, i}}{W_{f, i}}$ is then computed and exhibited on the VSI EWMA-RZ control chart. For this example, the following assumptions are supposed:

- Both $W_{p, i, j}$ and $W_{f, i, j}$ can be well approximated by normal variables with constant coefficients of variation $\gamma_{p}=0.02$ and $\gamma_{f}=0.01$, respectively.

- The in-control correlation coefficient between these two variables is $\rho_{0}=0.8$.

- The shift size $\tau=1.01$ is anticipated. 


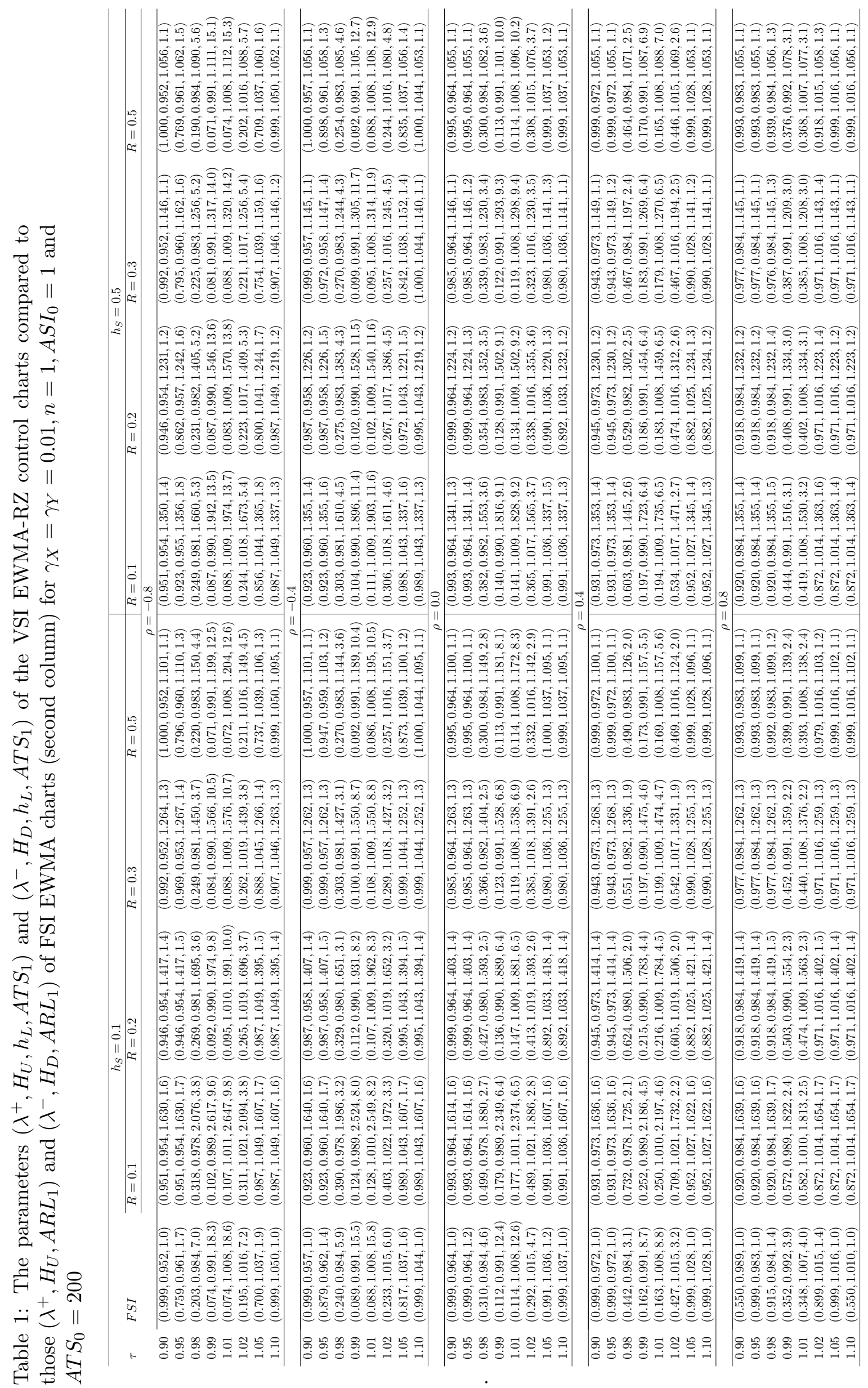









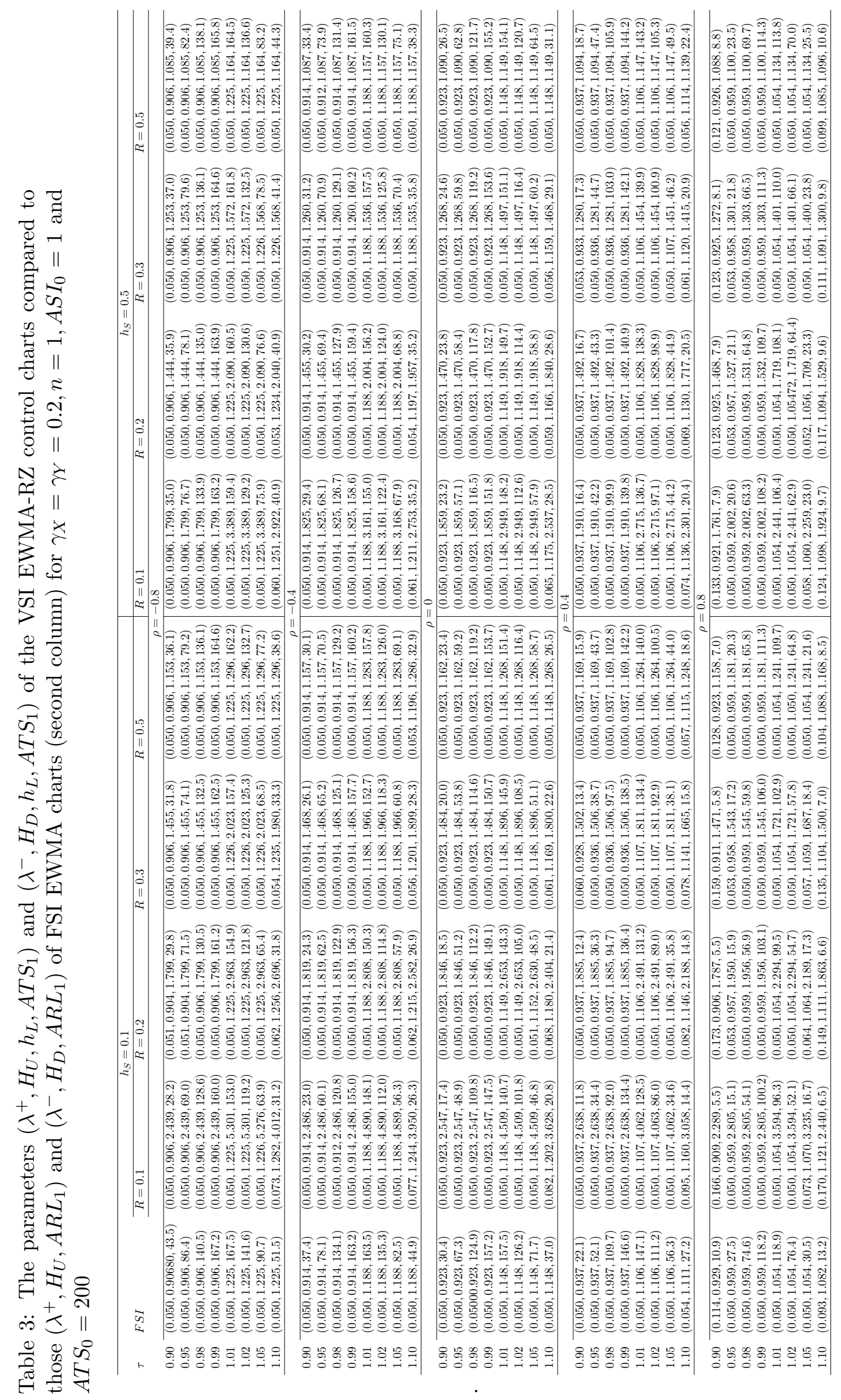




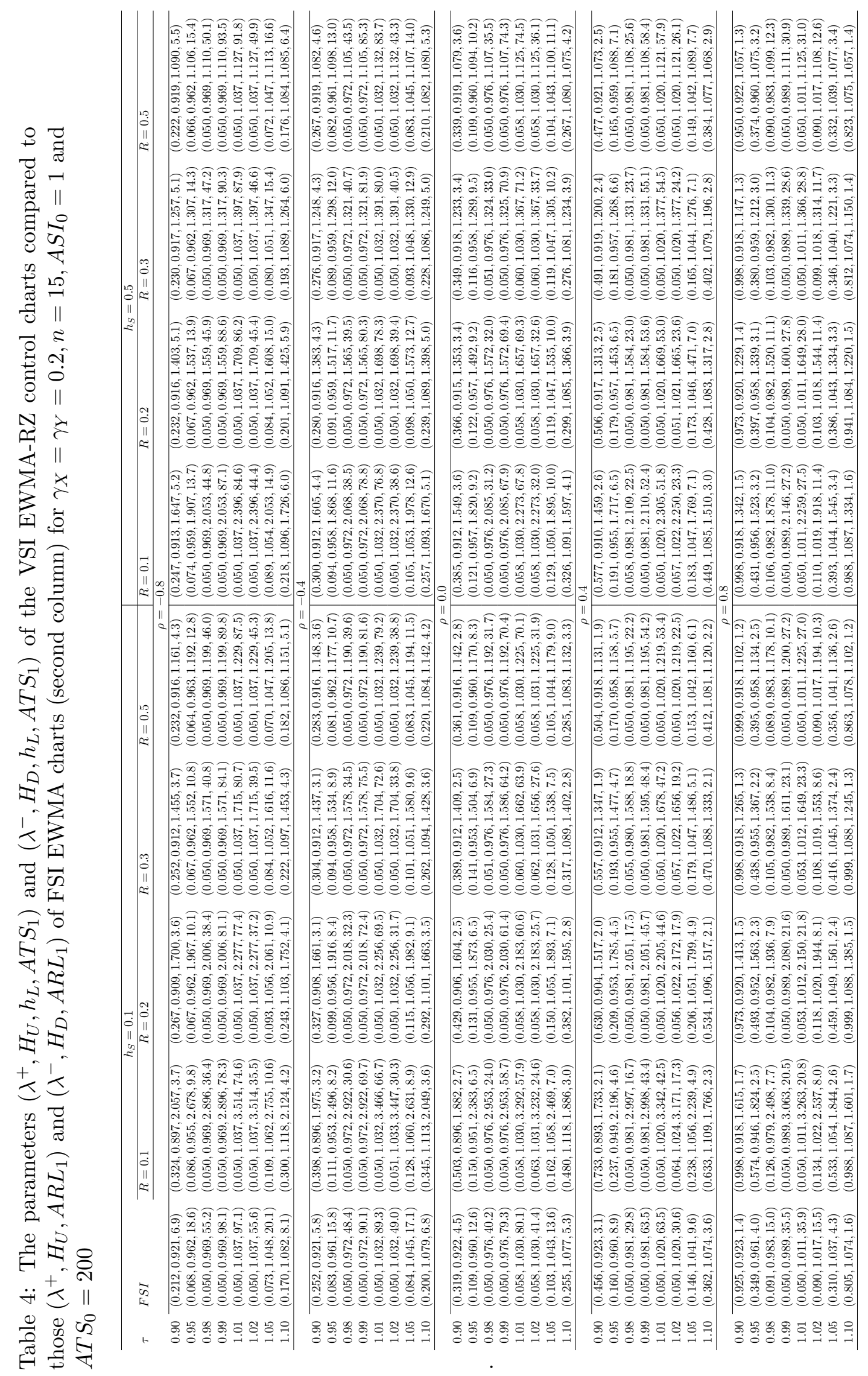




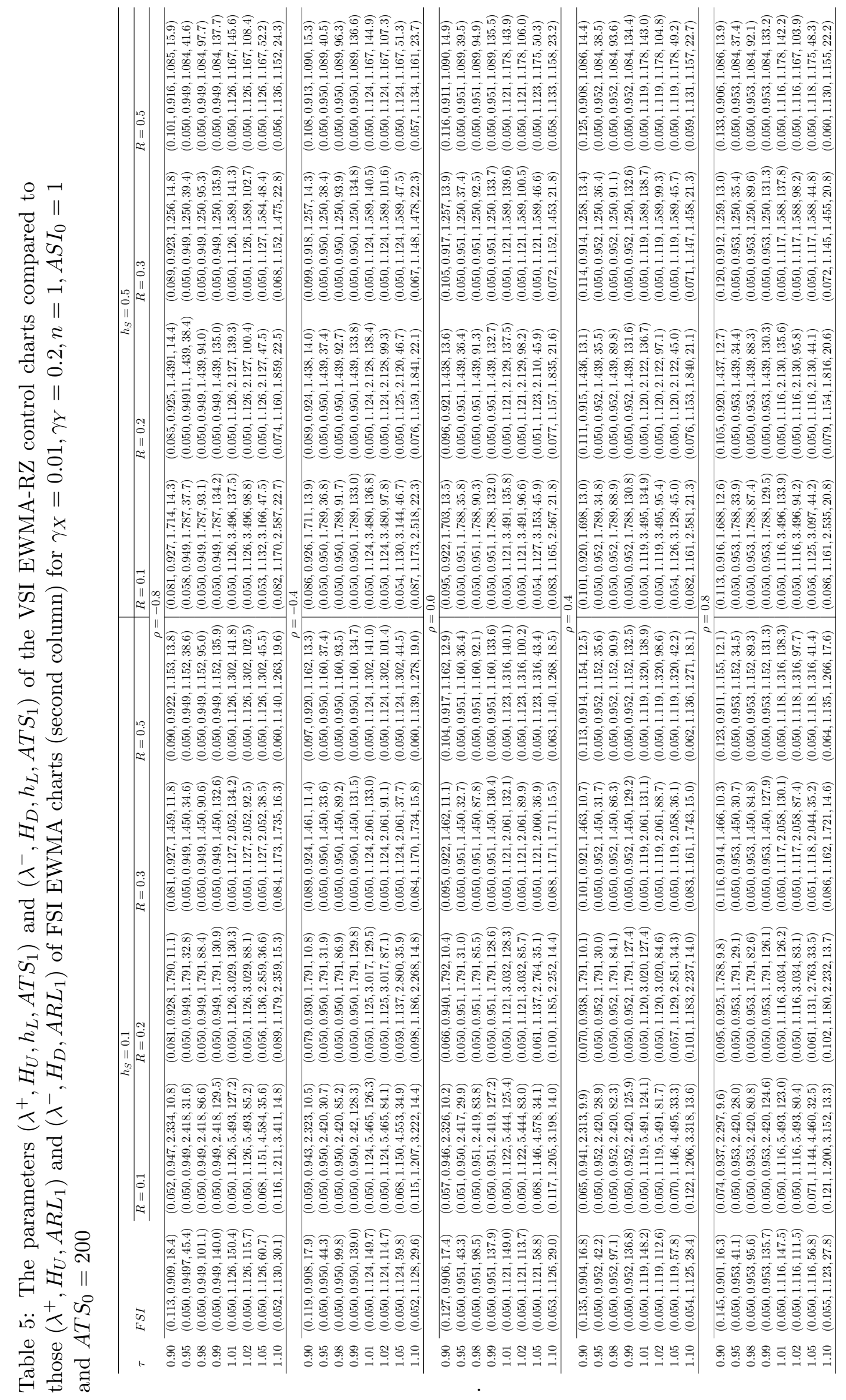




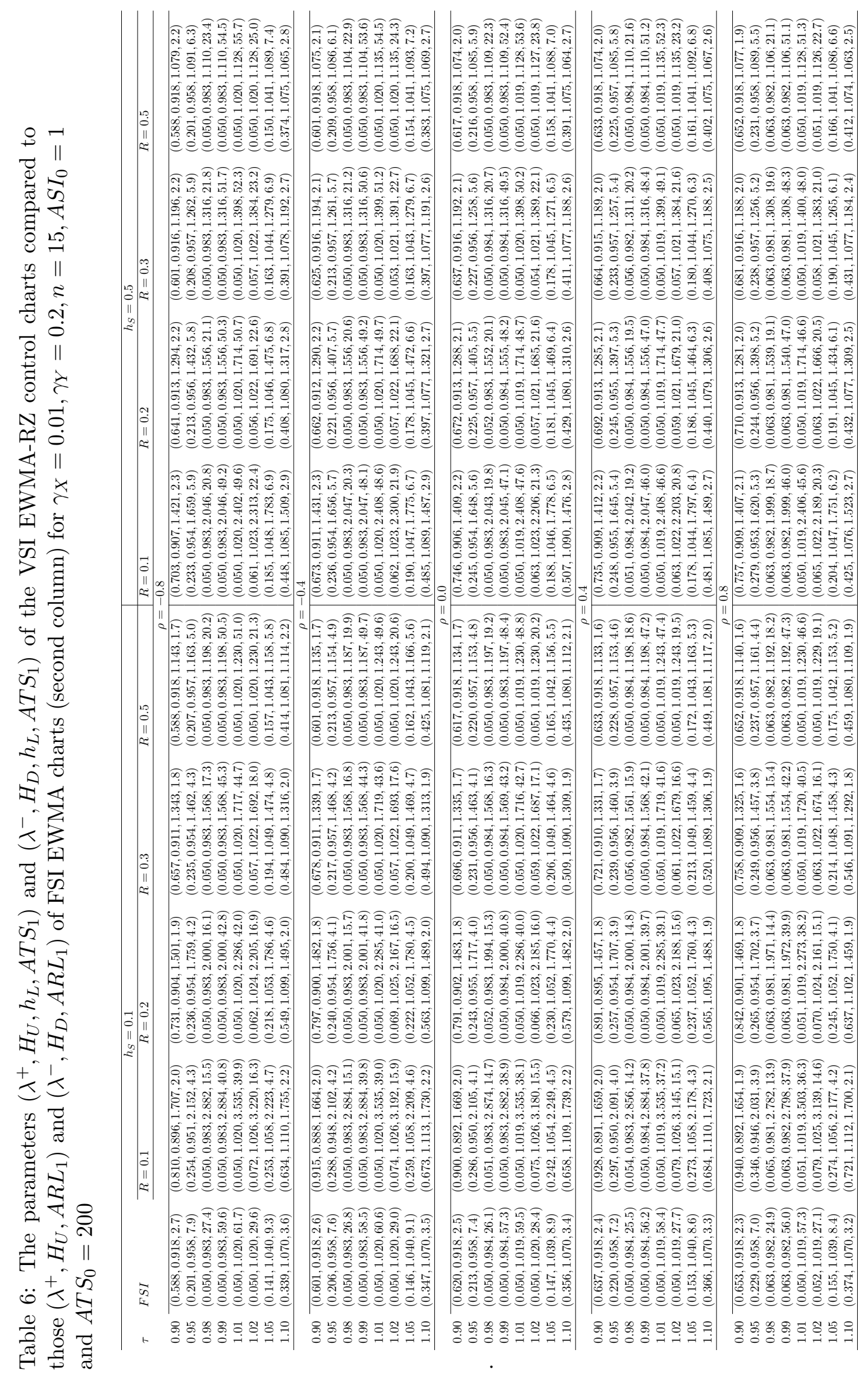




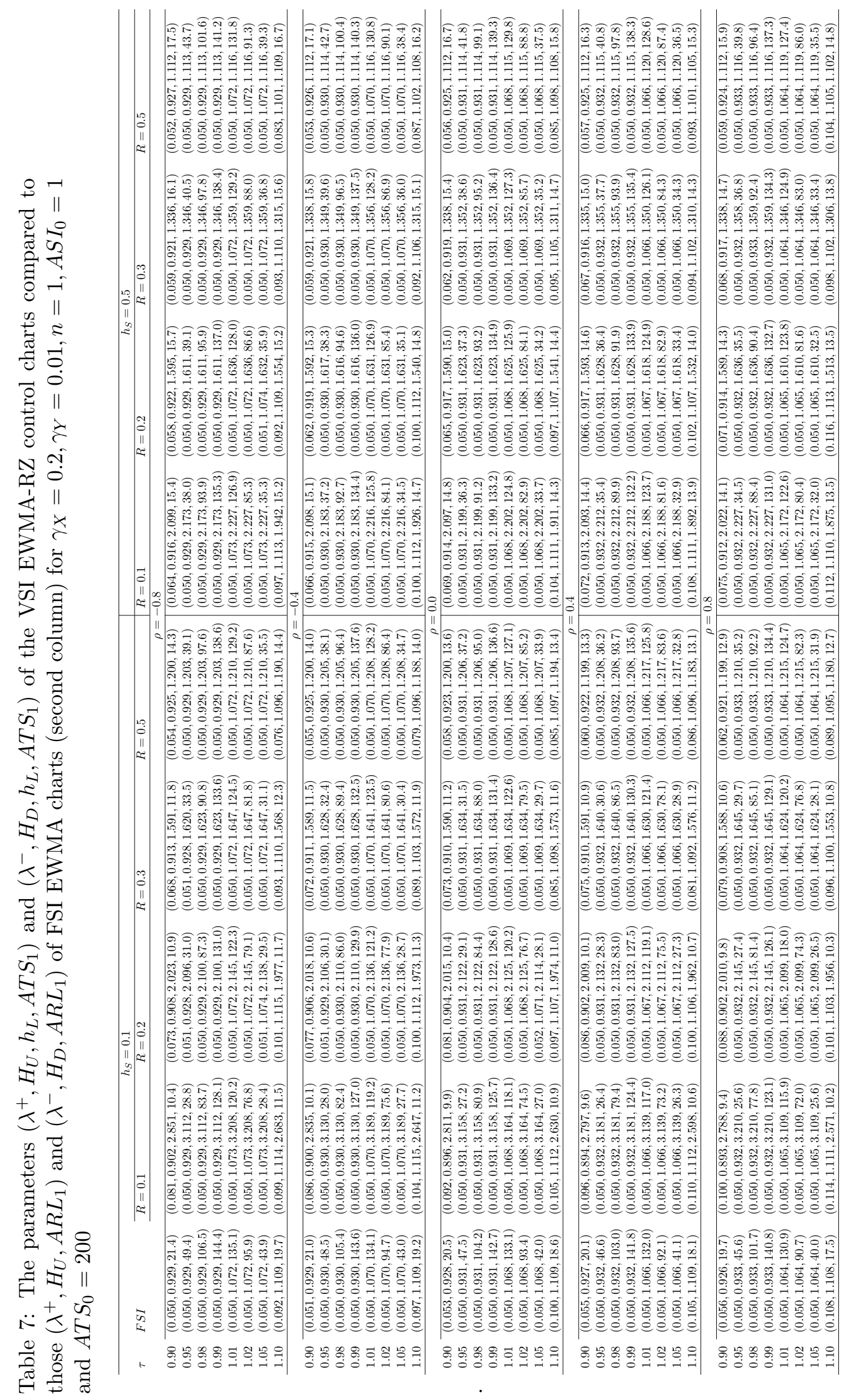




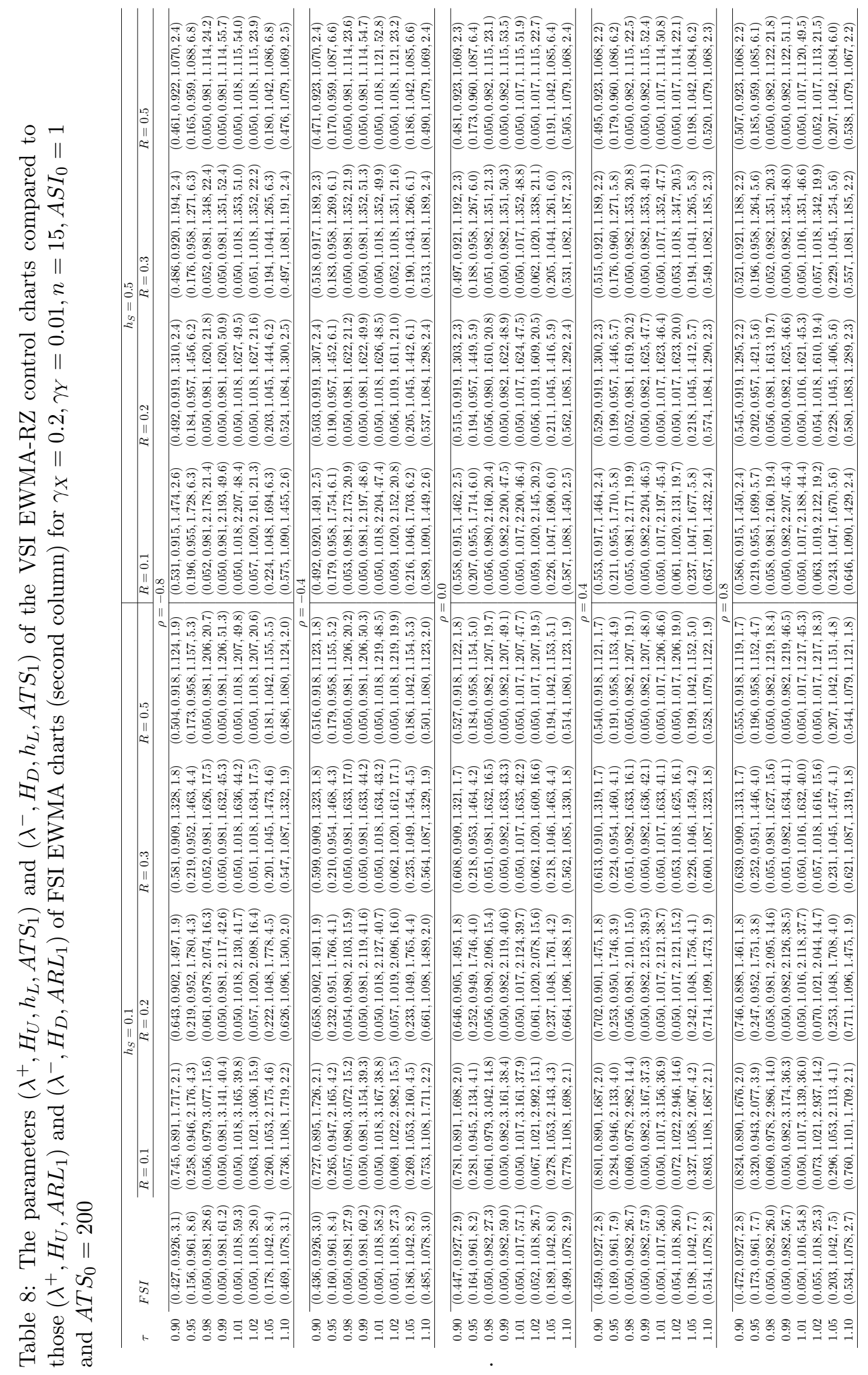




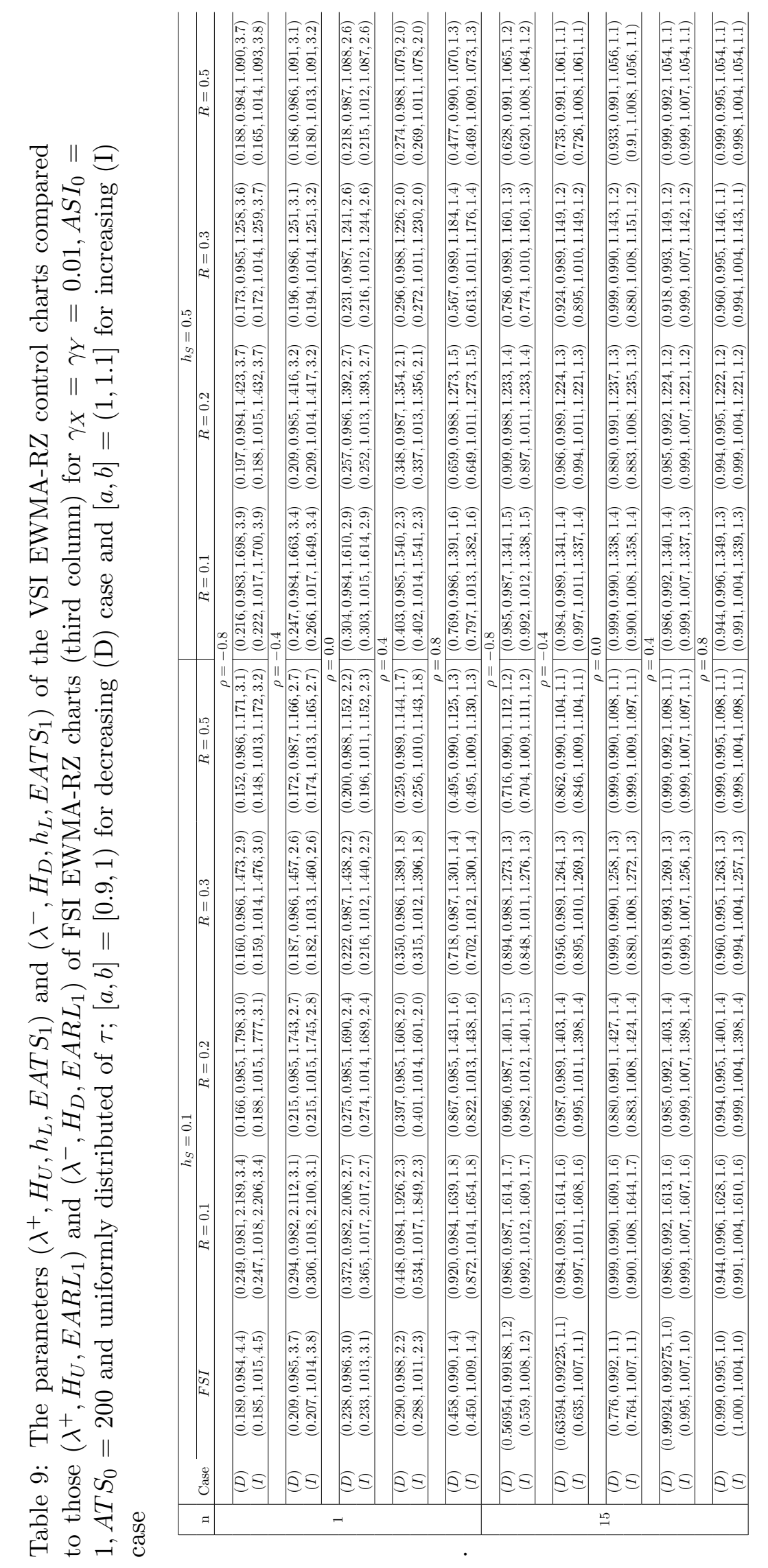




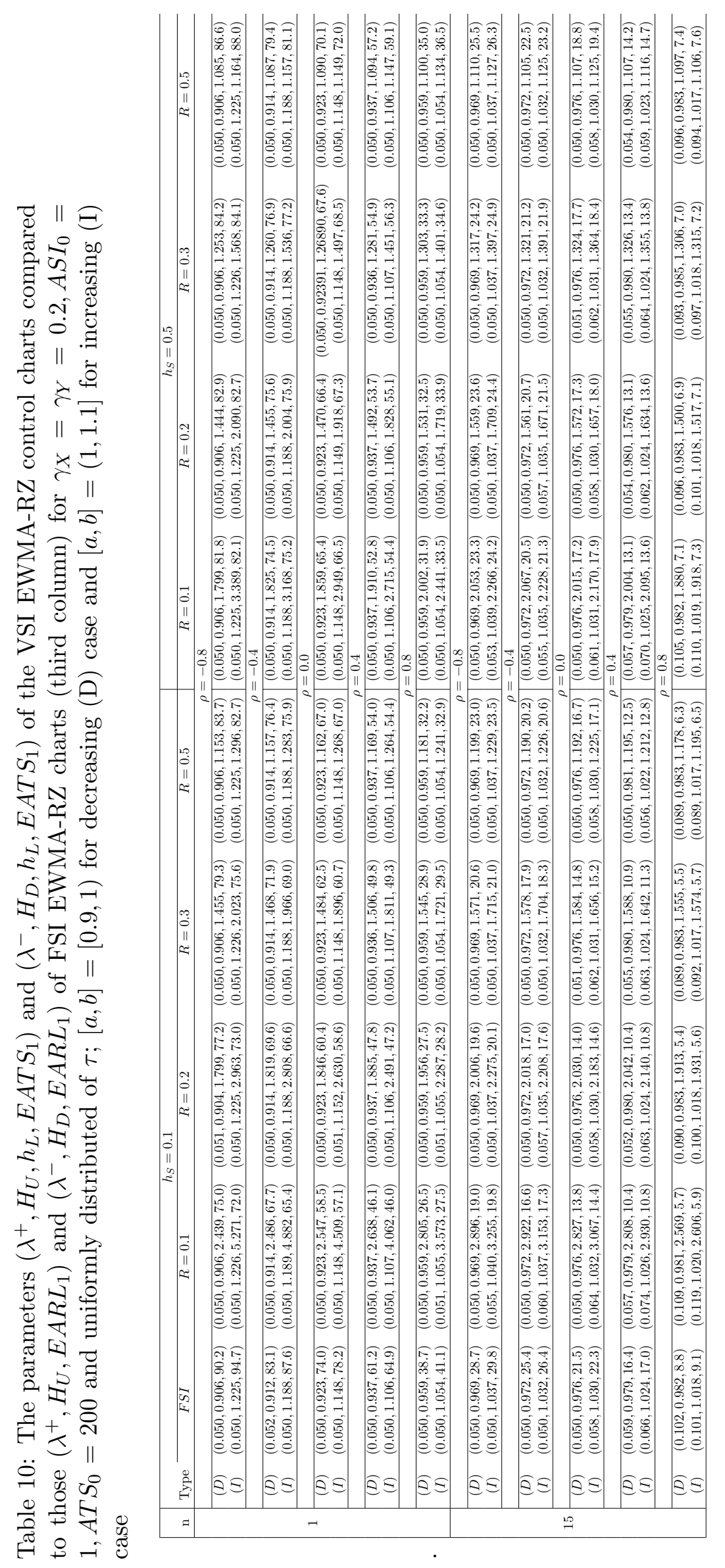




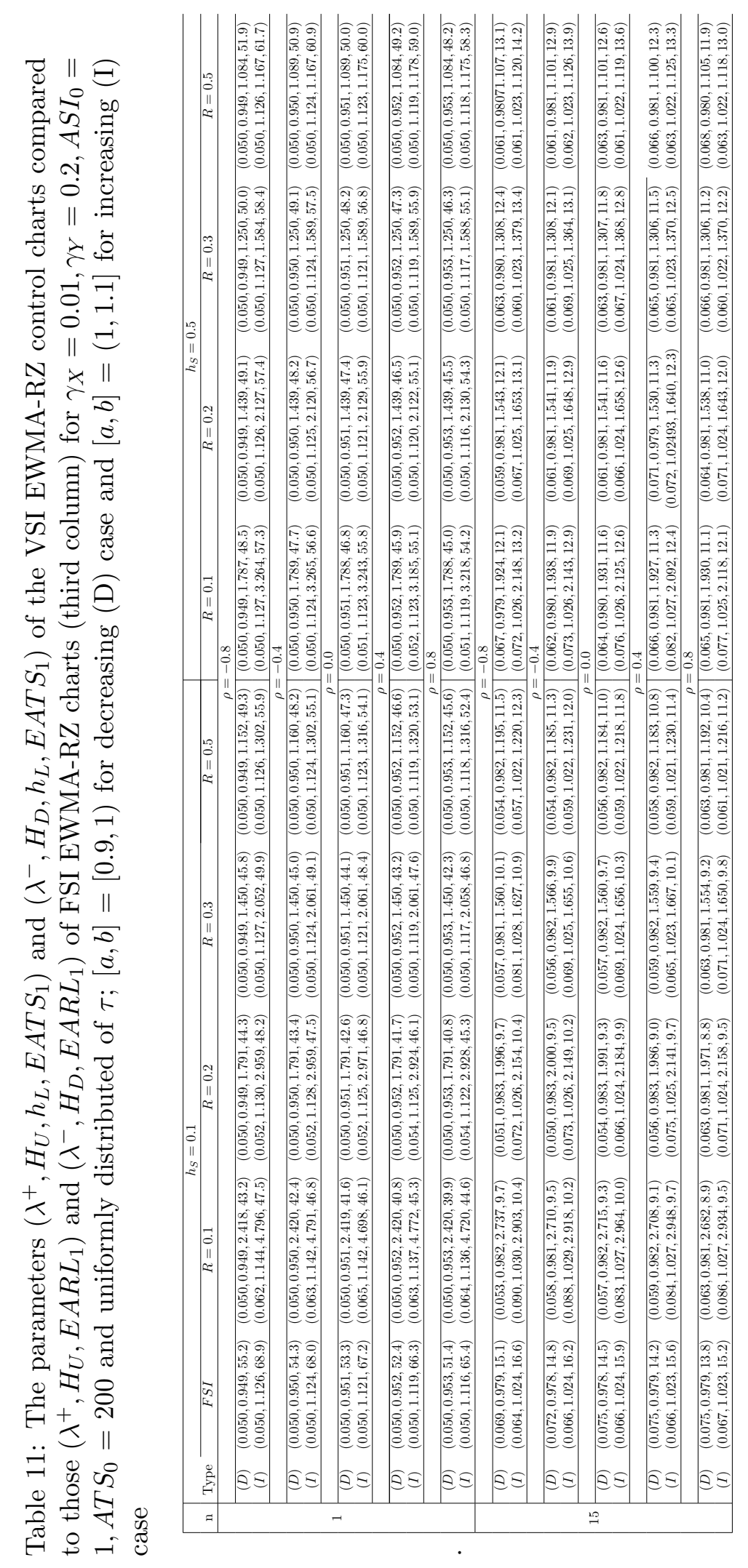




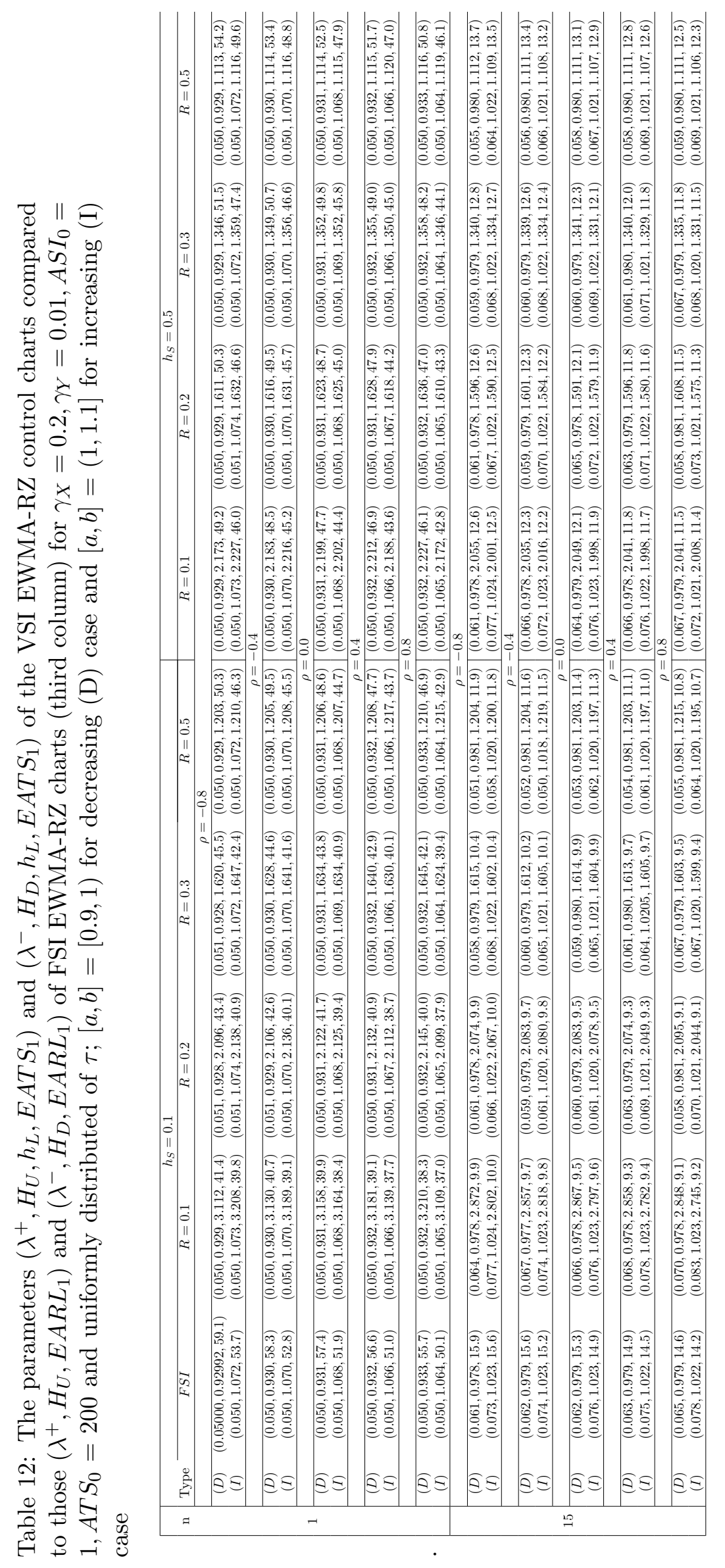


Figure 4: VSI EWMA-RZ control charts for the food industry example

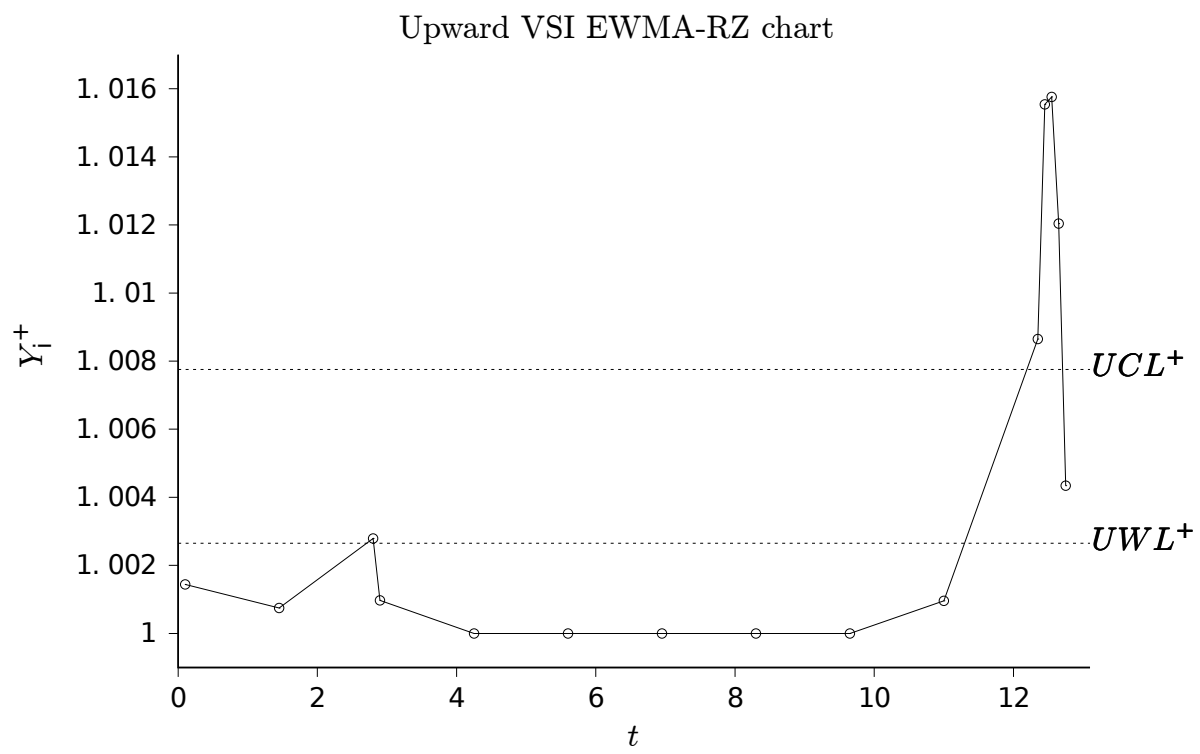

- The samling interval $h_{S}=0.1$ and the warning control coefficient $R_{U}=0.3$.

Given these values of parameters, we calculate $h_{L}=1.35, \lambda^{+}=0.4796970$, $H_{U}=1.0088404$ for optimal parameters of upward VSI EWMA-RZ control chart. The control limit and warning limit are then $U C L^{+}=1.0088404$ and $U W L^{+}=1.0026521$.

The set of collected data, the sample ratio $\hat{Z}_{i}$ and the control statistics $Y_{i}^{+}$are shown in Table 13. The obtained result shows that the process run in-control up to sample \#10. The upward VSI EWMA-RZ control chart signals the occurrence of the out-of-control condition in Figure 4 by plotting point \#12 above the control limit $U C L^{+}=1.007754$. This result is also boldfaced in Table 13. After correction actions to restore the process back to the in-control condition, the process is enabled to continue after sample $\# 14$.

\section{Concluding remarks}

This study has presented the optimal statistical design of the VSI EWMA control chart for monitoring the ratio of two normal random variables. We found that the new warning coefficient have significant impact on the proposed chart. In general, reducing the value of this coefficient is an effective 


\begin{tabular}{|c|c|c|c|c|c|c|c|c|c|c|c|}
\hline \multirow[b]{2}{*}{ Sample } & \multirow[b]{2}{*}{ SI } & \multicolumn{3}{|c|}{$W_{p, i, j}[\mathrm{gr}]$} & \multicolumn{4}{|c|}{$\bar{W}_{p, i}[\mathrm{gr}]$} & \multirow[b]{2}{*}{$\bar{W}_{f, i}[\mathrm{gr}]$} & \multirow[b]{2}{*}{$\hat{Z}_{i}=\frac{\bar{W}_{p, i}}{\bar{W}_{f, i}}$} & \multirow[b]{2}{*}{$Y_{i}^{+}$} \\
\hline & & Total time & Box Size & & & $W_{f, i, j}[\mathrm{gr}]$ & & & & & \\
\hline \multirow[t]{2}{*}{1} & 0.1 & 0.1 & $250 \mathrm{gr}$ & 25.479 & 25.355 & 24.027 & 25.792 & 24.960 & 25.122 & 1.003 & 1.00118 \\
\hline & & & & 25.218 & 25.171 & 24.684 & 25.052 & 25.107 & 25.046 & & \\
\hline \multirow[t]{2}{*}{2} & 1.35 & 1.45 & $250 \mathrm{gr}$ & 25.359 & 25.172 & 24.508 & 25.292 & 24.449 & 24.956 & 1.003 & 1.00072 \\
\hline & & & & 25.211 & 25.115 & 24.679 & 24.933 & 24.831 & 24.954 & & \\
\hline \multirow[t]{2}{*}{3} & 1.35 & 2.8 & $250 \mathrm{gr}$ & 24.574 & 24.864 & 25.865 & 25.107 & 24.811 & 25.044 & 1.005 & 1.00240 \\
\hline & & & & 24.784 & 24.868 & 25.377 & 24.879 & 24.734 & 24.929 & & \\
\hline \multirow[t]{2}{*}{4} & 0.1 & 2.9 & $250 \mathrm{gr}$ & 25.313 & 24.483 & 24.088 & 25.184 & 25.681 & 24.950 & 0.999 & 1.00106 \\
\hline & & & & 25.338 & 24.859 & 24.305 & 25.115 & 25.251 & 24.974 & & \\
\hline \multirow[t]{2}{*}{5} & 1.35 & 4.25 & $250 \mathrm{gr}$ & 25.557 & 24.959 & 25.023 & 24.482 & 25.531 & 25.111 & 0.998 & 1.00000 \\
\hline & & & & 25.277 & 25.402 & 25.012 & 24.937 & 25.148 & 25.163 & & \\
\hline \multirow[t]{2}{*}{6} & 1.35 & 5.6 & $250 \mathrm{gr}$ & 24.882 & 24.473 & 24.814 & 25.418 & 24.732 & 24.864 & 0.997 & 1.00000 \\
\hline & & & & 24.962 & 24.644 & 24.817 & 25.419 & 24.818 & 24.932 & & \\
\hline \multirow[t]{2}{*}{7} & 1.35 & 6.95 & $500 \mathrm{gr}$ & 49.848 & 48.685 & 49.994 & 49.910 & 49.374 & 49.562 & 0.999 & 1.00000 \\
\hline & & & & 49.993 & 49.128 & 49.830 & 49.566 & 49.422 & 49.588 & & \\
\hline \multirow[t]{2}{*}{8} & 1.35 & 8.3 & $500 \mathrm{gr}$ & 49.668 & 50.338 & 49.149 & 47.807 & 49.064 & 49.205 & 0.990 & 1.00000 \\
\hline & & & & 49.695 & 50.681 & 49.640 & 48.969 & 49.612 & 49.720 & & \\
\hline \multirow[t]{2}{*}{9} & 1.35 & 9.65 & $500 \mathrm{gr}$ & 51.273 & 48.303 & 48.510 & 50.594 & 48.591 & 49.454 & 0.993 & 1.00000 \\
\hline & & & & 50.366 & 49.210 & 49.844 & 49.890 & 49.595 & 49.781 & & \\
\hline \multirow[t]{2}{*}{10} & 1.35 & 11 & $500 \mathrm{gr}$ & 48.720 & 51.566 & 49.677 & 50.651 & 50.344 & 50.192 & 1.002 & 1.00079 \\
\hline & & & & 49.721 & 50.215 & 50.178 & 50.324 & 50.071 & 50.102 & & \\
\hline \multirow[t]{2}{*}{11} & 1.35 & 12.35 & $500 \mathrm{gr}$ & 51.372 & 51.700 & 51.000 & 50.886 & 49.641 & 50.920 & 1.017 & 1.00717 \\
\hline & & & & 50.164 & 50.272 & 49.884 & 50.061 & 49.845 & 50.045 & & \\
\hline \multirow[t]{2}{*}{12} & 0.1 & 12.45 & $500 \mathrm{gr}$ & 52.020 & 53.182 & 51.374 & 51.342 & 48.771 & 51.138 & 1.023 & 1.01340 \\
\hline & & & & 50.749 & 50.369 & 49.697 & 49.575 & 49.440 & 49.966 & & \\
\hline \multirow[t]{2}{*}{13} & 0.1 & 12.55 & $500 \mathrm{gr}$ & 52.360 & 49.412 & 50.704 & 50.370 & 50.901 & 50.949 & 1.016 & 1.01443 \\
\hline & & & & 50.047 & 49.981 & 50.297 & 50.408 & 50.026 & 50.152 & & \\
\hline \multirow[t]{2}{*}{14} & 0.1 & 12.65 & $500 \mathrm{gr}$ & 52.498 & 50.447 & 48.713 & 48.574 & 50.275 & 50.101 & 1.008 & 1.01190 \\
\hline & & & & 50.064 & 50.124 & 49.162 & 48.865 & 50.344 & 49.712 & & \\
\hline \multirow[t]{2}{*}{15} & 0.1 & 12.75 & $250 \mathrm{gr}$ & 25.123 & 24.658 & 24.468 & 25.030 & 25.071 & 24.870 & 0.996 & 1.00564 \\
\hline & & & & 25.041 & 24.790 & 24.835 & 25.211 & 25.008 & 24.977 & & \\
\hline
\end{tabular}

Table 13: The food industry example data 
solution to enhance the performance of the VSI EWMA-RZ control chart, but in the case both two variables have small coefficient of variation, the charts power is somewhat better with $R_{U}$ or $R_{D}$ around 0.3 . The influence of other process parameters on the chart are also investigated for better understanding of this new type of control chart. From the numerical comparison, we prove that our proposed control charts are better than the standard EWMA-RZ control chart in detecting the ratio shifts.

\section{References}

[1] P. Castagliola, G. Celano, S. Fichera, and F. Giuffrida. A variable sampling interval $s^{2}$-ewma control chart for monitoring the process variance. International Journal of Technology Management, 37(1-2): 125-146, 2006.

[2] P. Castagliola, P. E. Maravelakis, and F. O. Figueiredo. The EWMA Median chart with estimated parameters. IIE Transactions, 48(1):6674, 2016.

[3] W.C. Yeong, M.B.C. Khoo, L.K. Teoh, and M.A. Rahim. Monitoring the coefficient of variation using a variable sampling interval ewma chart. Journal of Quality Technology, 49(3):380-401, 2017.

[4] P. Castagliola, A. Amdouni, H. Taleb, and G. Celano. Monitoring the coefficient of variation using ewma charts. Journal of Quality Technology, 43(3):249-265, 2011.

[5] P. Castagliola, A. Achouri, H. Taleb, G. Celano, and S. Psarakis. Monitoring the coefficient of variation using a variable sampling interval control chart. Quality and Reliability Engineering International, 29(8): 1135-1149, 2013.

[6] K.W. Linna and W.H. Woodall. Effect of measurement error on Shewhart control chart. Journal of Quality Technology, 33(2):213-222, 2001.

[7] G. Tagaras. A survey of recent developments in the design of adaptive control charts. Journal of Quality Technology, 30(3):212-223, 1988.

[8] G. Celano, P. Castagliola, A. Faraz, and S. Fichera. Statistical Performance of a Control Chart for Individual Observations Monitoring the Ratio of two Normal Variables. Quality and Reliability Engineering International, 30(8):1361-1377, 2014.

[9] G. Celano and P. Castagliola. A Synthetic Control Chart for Monitoring the Ratio of Two Normal Variables. Quality and Reliability Engineering International, 32(2):681-696, 2016. 
[10] K.P. Tran, P. Castagliola, and G. Celano. Monitoring the Ratio of Two Normal Variables Using Run Rules Type Control Charts. International Journal of Production Research, 54(6):1670-1688, 2016.

[11] K.P. Tran, P. Castagliola, and G. Celano. Monitoring the Ratio of Population Means of a Bivariate Normal distribution using CUSUM Type Control Charts. Statistical Papers, 2016. In press, DOI: 10.1007/s00362016-0769-4.

[12] D. Öksoy, E. Boulos, and L.D. Pye. Statistical Process Control by the Quotient of two Correlated Normal Variables. Quality Engineering, 6 (2):179-194, 1994.

[13] G. Celano and P. Castagliola. Design of a phase II Control Chart for Monitoring the Ratio of two Normal Variables. Quality and Reliability Engineering International, 32(1):291-308, 2016.

[14] K.P. Tran, P. Castagliola, and G. Celano. Monitoring the Ratio of Two Normal Variables Using EWMA Type Control Charts. Quality and Reliability Engineering International, 32(2):1853-1869, 2016.

[15] K.P. Tran and S. Knoth. Steady-state arl analysis of arl-unbiased ewmarz control chart monitoring the ratio of two normal variables. Qual Reliab Eng Int., pages 1-14, 2018.

[16] K.P. Tran, P. Castagliola, and G. Celano. The performance of the Shewhart-RZ control chart in the presence of measurement error. International Journal of Production Research, 54:7504-7522, 2016.

[17] K.P. Tran, P. Castagliola, G. Celano, and M.B.C. Khoo. Monitoring compositional data using multivariate exponentially weighted moving average scheme. Quality and Reliability Engineering International, 34 (3):391-402, 2018. doi: https://doi.org/10.1002/qre.2260.

[18] R.C. Geary. The Frequency Distribution of the Quotient of Two Normal Variates. Journal of the Royal Statistical Society, 93(3):442-446, 1930.

[19] J. Hayya, D. Armstrong, and N. Gressis. A note on the Ratio of Two Normally Distributed Variables. Management Science, 21(11):13381341, 1975.

[20] A. Cedilnik, K. Kosmelj, and A. Blejec. The Distribution of the Ratio of Jointly Normal Variables. Metodoloski Zvezki, 1(1):99-108, 2004.

[21] T. Pham-Gia, N. Turkkan, and E. Marchand. Density of the ratio of two normal random variables and applications. Communications in Statistics - Theory and Methods, 35(9):1569-1591, 2006. 
[22] D. Brook and D.A. Evans. An approach to the probability distribution of CUSUM run length. Biometrika, 59(3):539-549, 1972.

[23] M.S. Saccucci, W.A. Raid, and J.M. Lucas. Exponentially weighted moving average control schemes with variable sampling intervals. Commun. Statist-Simula, 21(3):627-657, 1992.

[24] M.R Reynolds and J.C. Arnold. Optimal one-sided shewhart charts with variable sampling interval. Sequential Analysis, 80(1):181-192, 1989. 